\title{
Outcomes of Swallowing Rehabilitation in Patients with Dysphagia: Retrospective Study
}

\author{
https://doi.org/10.47210/bjohns.2020.v28i2.334
}

\section{Anindita Arun Banik}

\section{$\underline{\text { Introduction }}$}

ABSTRACT

Swallowing, as the first phase of digestion, is one of the most complicated neuromuscular processes of the central nervous system. The presence of dysphagia can itself cause medical, psychosocial, and economic complications. A medical complication of dysphagia includes aspiration pneumonia, malnutrition, significant weight loss, and dehydration.

\section{Materials and Methods}

It was a retrospective study. In information regarding speech and swallowing therapeutics were studied from the routine clinical records by speech pathologist.

\section{$\underline{\text { Results }}$}

The present retrospective study was focused on highlighting the outcomes of swallowing rehabilitation in patients with dysphagia in various aetiologies. It was observed that swallowing compensatory strategies, postural changes, rehabilitative manoeuvres, thermal stimulation, indirect and direct therapy showed tremendous improvement in patients with dysphagia with various aetiologies.

Conclusion

This study provides further information regarding the severity of swallowing physiology in disorders and its improvement subsequent to traditional swallowing therapy which will contribute to literature in Indian context.

$\underline{\text { Keywords }}$

Deglutition Disorders; Rehabilitation; Outcomes

$O$ wallowing, as the first phase of digestion, is one of the most complicated neuromuscular processes of the central nervous system. It involves multiple areas of the brain and a series of voluntary and involuntary muscular contractions. Oropharyngeal dysphagia is a highly prevalent clinical condition among stroke patients, but the prevalence of dysphagia is different in various studies, because of differences in the definition of dysphagia, the method of assessing swallowing function, the timing of swallowing assessment after stroke, and the number and type of stroke patients studied. ${ }^{1,2}$ Overall, swallowing disorders (dysphagia with or without aspiration) are seen in about half (55\%) of all stroke patients admitted to hospital. ${ }^{3,4}$

A medical complication of dysphagia includes aspiration pneumonia, malnutrition, significant weight loss, and dehydration ${ }^{5}$ along with psychosocial because eating is a pleasurable and social activity, and inability to eat normally may affect patient morale and quality-oflife.6 Complications due to dysphagia especially include pneumonia, and managing infection also increases healthcare costs by increasing the length of hospital stay and increasing the need for expensive respiratory and nutritional support. ${ }^{3,4}$

To prevent and minimize these complications, diagnosis and management of dysphagia must be done

1 - Consultant Audiologist and speech pathologist, Holy

Family Hospital and Medical Research Centre, Mumbai

\section{Corresponding author:}

Ms. Anindita Arun Banik

email: aninditabanik20@gmail.com 
as soon as possible by a speech-language pathologist. ${ }^{5}$ Various rehabilitation techniques are utilized by a speech pathologist for management dysphagia. The traditional swallowing therapy includes Compensatory strategies, postural changes, rehabilitative manoeuvres, thermal stimulation, indirect and direct therapy. ${ }^{6}$

Compensatory medical approaches include: enteral feeding by means of a nasogastric tube or by percutaneous endoscopic gastrostomy, modification of food consistency, postural correction to facilitate bolus transition, reducing rate of eating and ensuring oral hygiene by conventional oral care. ${ }^{7,8}$

Other approaches are rehabilitative methods, including oral motor exercises; airway-protecting maneuver, thermal-tactile stimulation, and Shaker exercises. ${ }^{6-10}$ Recently, neuromuscular electrical stimulation, biofeedback, and transcranial magnetic stimulation have been used as techniques for swallowing therapy. ${ }^{11}$

Some studies have only focused on early intervention, and do not consider the time at which swallowing rehabilitation should be initiated for optimal recovery. ${ }^{12-15}$ Current state of the art in the management of dysphagia includes a wide variety of multidisciplinary practices. These practices range from simple diet adjustment to more invasive surgical interventions, depending on the nature and severity of the disorder. Because management of dysphagia is a relatively new specialization, the direct therapeutic interventions offered by the speech language pathologist have consisted largely of compensatory mechanisms by which the patient compensates for disordered swallowing with adjustments in diet consistency or behaviorally based manoeuvres.

The role of speech language pathologist is significant in the rehabilitation of swallowing disorders. It is the need at present to emphasize the importance of traditional therapy which helps in improvement in swallowing mechanism. There are a lot of studies presented in the western context emphasizing on swallowing therapy. Hence, there arises a need to focus on the same in Indian context. The present retrospective study documents outcomes of swallowing rehabilitation in a group of patients with dysphagia. This study provides further information regarding the severity of swallowing physiology in disorders and its improvement subsequent to traditional swallowing therapy which will contribute to literature in Indian context. Hence, the aim of the study is to evaluate the outcomes in swallowing rehabilitation in the patients with dysphagia.

\section{Materials and Methods}

It was a retrospective study. This has been approved by the Ethics committee of the hospital.

The subjects of this retrospective study observational study included adults and children from the age groups of 0 to 80 years. These were divided as follows: Group I- 0 to 10 years, Group II- 10 to 20 years, Group III- 21 to 40 years, Group IV- 41 to 60 years, Group V- 61 to 80 years. Individuals reporting or referred to the department of Audiology and Speech Pathology with neurological speech, language swallowing disorders, articulation, fluency, voice disorders for therapy were studied. The Clinical speech language swallowing proforma was used to evaluate patients and to diagnose the patients for speech language swallowing disorders. The proforma was to assess basically the above mentioned domains and for assessing the severity of dysphagia. Nair hospital bedside assessment and severity scale was used which is freely available for use. The severity is determined by adding all scores and the total score is compared with the severity scale. ${ }^{16}$

The information about the speech language and swallowing disorders therapeutics was considered for this study which was exclusively performed by the Speech Language Pathologist. The therapy outcomes of patients, who were diagnosed and referred by doctors from IPD and OPD of Neurology, Medicine, ENT, oncology and other departments of the hospital, were studied. This included patients from past 2 years (October 2016- October 2018) of speech language and swallowing therapy. The information based on therapeutics was studied in terms of the diagnosis, age groups gender, severity of dysphagia and rehabilitative outcomes. The severity of dysphagia was assessed pre and post therapy and compared. This further helped to determine if patients could be started with oral feeds and alternative mode of nutrition i.e. Ryle's Tube or Nasogastric Tube or PEG tube can be eliminated. The 


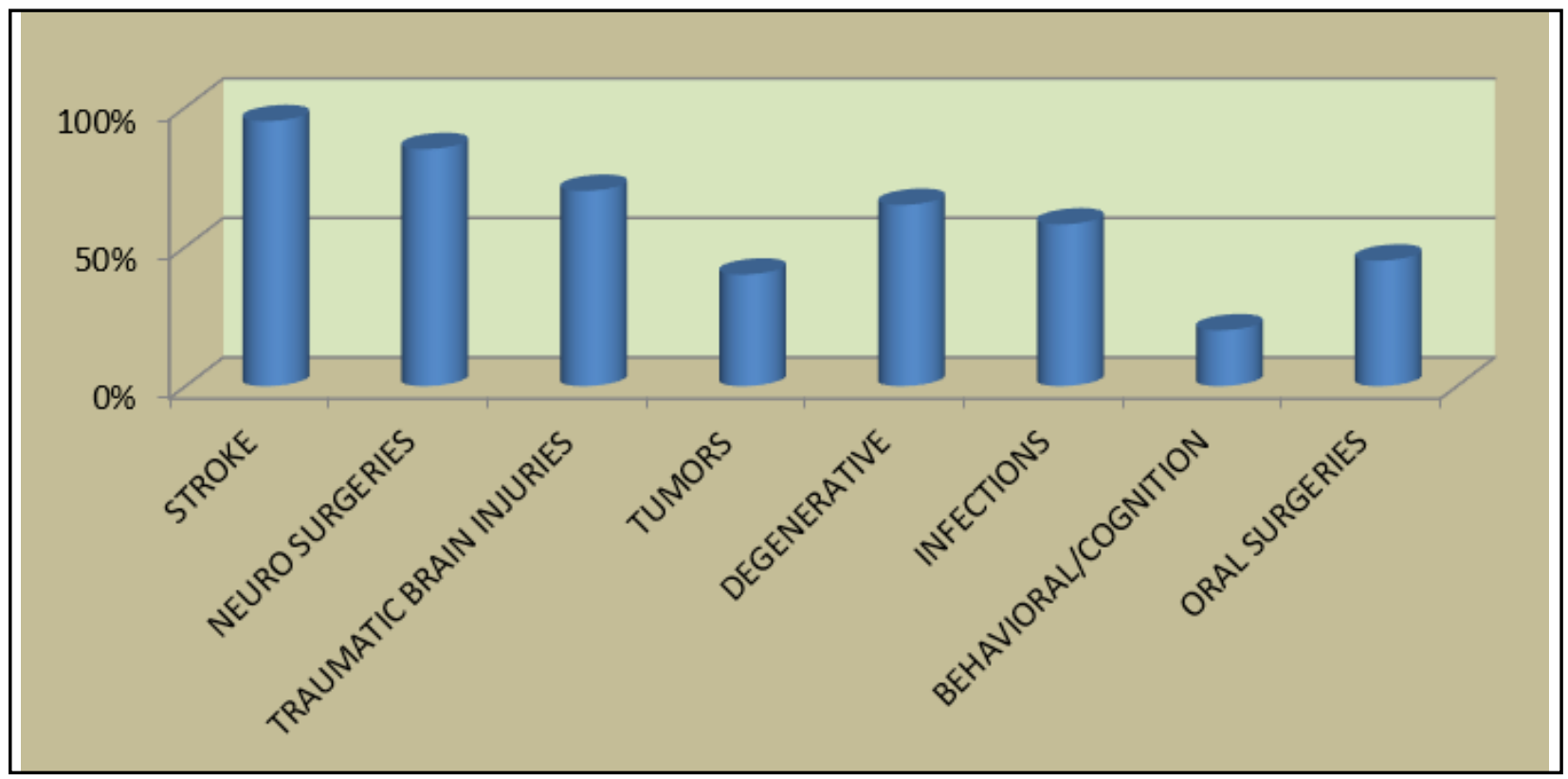

Fig. 1. Etiologies causing Dysphagia

analysis was done using percentage and non parametric tests Chi-square.

\section{Results}

All patients were individually evaluated as per the proforma and clinical swallowing, speech and language evaluation were done using bedside evaluation scales before initiating therapy. This study was based on outcomes of swallowing rehabilitation in patients with dysphagia in a tertiary care multidisplinary hospital. It was observed that the total number of dysphagia cases was 185 and sessions required were 690 to rehabilitate patients with dysphagia. This included various types of etiologies and severities of dysphagia. (Fig. 1) All individuals received therapy on one to one basis either bedside on or on OPD basis. The number of sessions for each individual was unequal depending on the recovery status. The recovery was based on the post therapy assessment using the severity scale of swallowing for each. (Table I) Those cases which were drop outs were not included in the study; rest were discharged based on recovery status.

The most common aetiology of dysphagia was stroke. (Fig. 1) Other causes of dysphagia were neurological surgeries, traumatic brain injuries, tumors, infections in central and peripheral nervous systems, degenerative neurogenic disorders, oral and neck surgeries and behavioral/cognitive etiologies. Behavioral or cognitive problems lead to lesser amount of dysphagia/ feeding issues. These etiologies can lead to dysphagia in 3 different phases i.e. oral preparatory, oral phase and pharyngeal phase.

Oral or head and neck surgeries due to oral cancers involving any oral structure i.e. tongue, buccal cavity, hard or soft palate, thyroid/parathyroid. Some patients

Table I: Pre and Post therapy Outcome measures

SEVERITY RANGE OF DYSPHAGIA

\begin{tabular}{|l|c|c|}
\hline & SEVERITY RANGE OF DYSPHAGIA & SCORES \\
\hline Pre therapy severity & Severe to complete & $5-6$ \\
\hline Post therapy severity & Normal to slight & $0-1$ \\
\hline
\end{tabular}




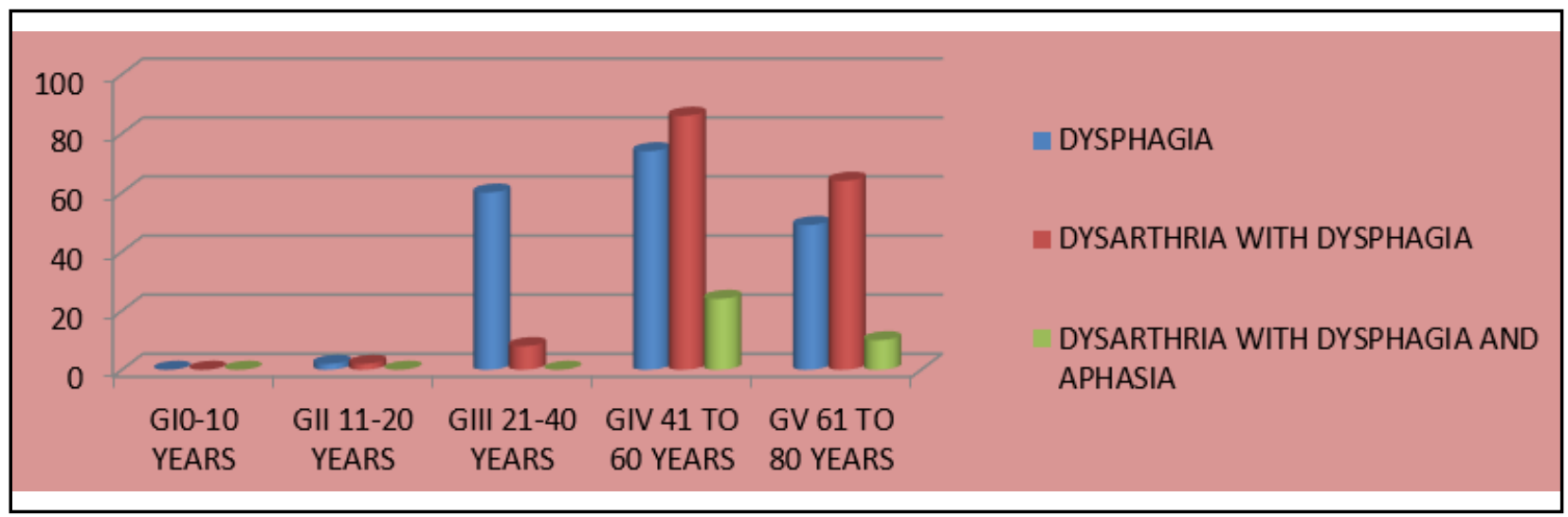

Fig. 2. Disorders as per age groups

undergoing mandibullectomy, glossectomy had oral preparatory phase dysphagia. Most of these etiologies are mechanical causes of dysphagia can affect the oral preparatory, oral and pharyngeal phase of swallow eg. Post thyroidectomy leading to vocal cord palsy. Few patients with Dementia had behavioral issues while feeding despite not having difficulty with swallowing mechanism. Most of the patients in the acute stage of dysphagia were on alternative mode of swallowing for nutrition i.e. through Nasogastric Tube/ Ryle's Tube; only few had Percutaneous Endoscopic Gastrostomy
When results were studied as per age groups it was seen there was significant difference (Chi square $<0.001$ ) in all groups, while no difference as per gender. It was observed (Fig. 2) that maximum cases had dysphagia in age groups more than 21 years to 80 years of age. In the age groups of 41 to 60 years and Group 61 to 80 years it was observed patients had dysphagia, and also had dysphagia with other disorders i.e. dysarthria and aphasia. With increasing age groups there was more occurrence of dysphagia with other neurocommunication and motor speech disorders. (PEG).

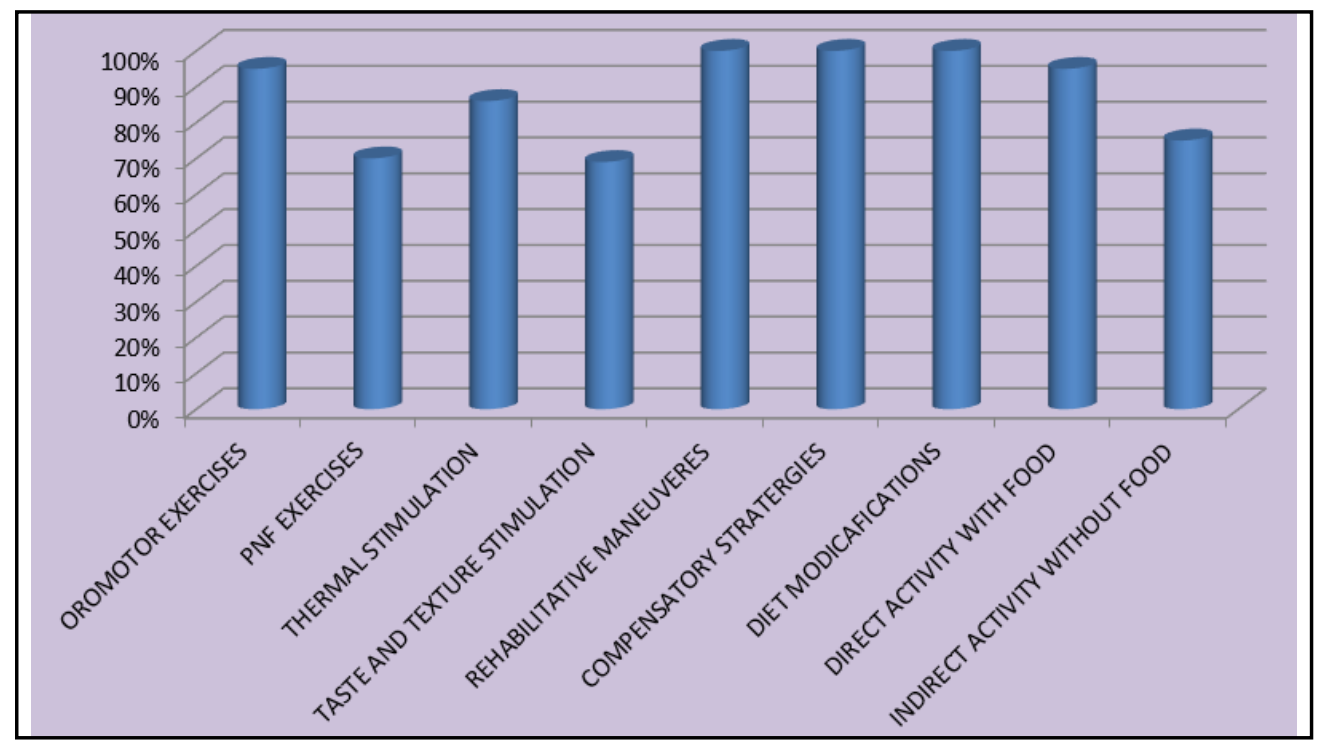

Fig. 3. Therapeutic Rehabilitative Management 
Various therapeutic management options are utilized by Speech Pathologist to rehabilitate patients with dysphagia. The current treatment of dysphagia in patients with stroke or other etiologies is the traditional swallowing therapy by a speech pathologist. Compensatory strategies, postural changes, rehabilitative maneuvers, thermal stimulation, indirect and direct therapy were included in this therapy. ${ }^{6}$ The therapeutic rehabilitative management included compensatory strategies, rehabilitative manoeuvres, thermal, taste and texture stimulation which is proprioceptive neuromuscular functioning exercises (PNF), oromotor exercises, diet modifications. All these were planned and used as indirect and direct methods in dysphagia rehabilitation. (Fig. 3)

\section{Discussion}

Rehabilitative maneuvers such as Masako, Guided Mendelson's, supraglottic and effortful swallow contributed almost $95 \%$ in rehabilitation. Compensatory strategies such as head positioning using chin tuck, chin up, head rotation to better side or head tilts were utilized as per different etiologies and phase of dysphagia. These behaviorally based maneuvers include techniques designed to improve airway protection, such as the Supraglottic swallow, Mendelson's, Masako and other manoeuvres designed to facilitate transfer of the bolus through the pharyngeal cavity by maximizing forces and minimizing resistance to the bolus, such as chintuck posturing. ${ }^{910}$ These were used in patients with neurological disorders such as cortical strokes, brainstem stokes like lateral meduallary syndrome, cerebellar strokes, progressive disorders such as Parkinson's disease, Progressive supranuclear palsy, Amyotropic lateral sclerosis etc. ${ }^{17,18}$

These interventions, although serving an important role, provide an immediate but only transient approach to the underlying physiologic deficit. These are compensatory in nature. Development of rehabilitative exercises and substantiation of efficacy are needed to address long-term changes in swallowing physiology. 19 Several techniques have been proposed as compensatory in intent, but clinical experience suggests that these techniques, when repeated in the context of an exercise regime, may facilitate overall change in swallowing physiology. The effortful swallow consists of a normal swallow in duration and sequence but with greater effort or force during the muscle contraction..$^{20-22}$

This technique was initially conceptualized to facilitate bolus clearance from pharyngeal recesses as a result of increased tongue-base approximation to the posterior pharyngeal wall. The Mendelsohn manoeuvre was designed to address deficits associated with reduced opening of the upper oesophageal sphincter. ${ }^{23-25}$ Execution involves the prolongation of a pharyngeal swallow when the thyroid cartilage is at its point of highest excursion, with the intent of maintaining opening of the upper oesophageal sphincter. ${ }^{23,24}$ More recently, a tongue-holding manoeuvre, or the Masako manoeuvre, was developed based on the observation that patients with base of tongue resection demonstrate spontaneous increase in posterior pharyngeal wall excursion to compensate for the base of the tongue. Execution of this manoeuvre significantly facilitates posterior pharyngeal wall movement and thus may provide a direct rehabilitative exercise to address pharyngeal contraction. ${ }^{26-29}$

With the use of various techniques in this study, eventually diet modification was done to initiate direct swallowing therapy. This was done using thin, thick liquids, semisolids blended, soft and hard solids consistencies of food. While doing so, patient's oral intake was monitored 3 times a day in proportions. Also while they were all on oral feeds for nutrition; symptoms of dysphagia was observed such as chocking, gurgle voice, breathing difficulties, aspiration, coughing during and after swallow. As they developed and could manage adequate oral feed quantities without any difficulties, they were recommended to have complete oral feed.

\section{Conclusion}

Through this study it was seen that there is a major role of a Speech Pathologist in dysphagia management in a hospital setup. This helps us to learn the effectiveness of traditional dysphagia therapy and it outcomes in various etiologies leading to dysphagia. This study also helps to create awareness amongst other medical fraternity 
about the role and importance to initiate therapeutic management at the earliest for patient benefits.

\section{References}

1. Martino R, Foley N, Bhogal S, Diamant N, Speechley M, Teasell R. Dysphagia after stroke: incidence, diagnosis, and pulmonary complications. Stroke 2005; 36 (12):2756-63

2. Mann G, Hankey GJ, Cameron D. Swallowing disorders following acute stroke: prevalence and diagnostic accuracy. Cerebrovasc Dis. 2000; 10(5):380-6

3. Langdon C, Blacker D. Dysphagia in stroke: a new solution. Stroke Res Treat. 2010;2010:570403. doi:10.4061/2010/570403

4. Langdon, PC, Lee AH, Binns CW. Dysphagia in acute ischaemic stroke: severity, recovery and relationship to stroke subtype. J Clin Neurosci. 2007; 14(7):630-4

5. Nilsson H, Ekberg O, Olsson R, Hindfelt B. Dysphagia in stroke: a prospective study of quantitative aspects of swallowing in dysphagic patients. Dysphagia 1998; 13(1):32-8

6. Vesey S. Dysphagia and quality of life. Br J Community Nurs 2013 (Suppl: S14, S16, S18-S14, S16, S19)

7. Davis LA. Quality of Life Issues Related to Dysphagia Topics in Geriatric Rehabilitation 2007; 23(4):352-65. doi: 10.4172/2329-9096.1000340

8. Murry T, Carrau RL. Clinical Management of Swallowing Disorders. 3rd ed. San Diego, CA: Plural Pub; 2012

9. Speyer R, Baijens L, Heijnen M, Zwijnenberg I. Effects of therapy in oropharyngeal dysphagia by speech and language therapists: a systematic review. Dysphagia 2010; 25(1):40-65

10. Logemann JL. Management of the patient with disordered oral feeding. In: Logemann JA, Ed: Evaluation and Treatment of Swallowing Disorders. Austin: Pro-Ed, 1983, 127-59

11. Huckabee ML. Outcomes of Swallowing Rehabilitation in Chronic Brainstem Dysphagia: A Retrospective Evaluation. Dysphagia 1999; 14:93-109

12. Khedr EM, Abo-Elfetoh N. Therapeutic role of rTMS on recovery of dysphagia in patients with lateral medullary syndrome and brainstem infarction. J Neurol Neurosurg Psychiatry 2010; 81(5):495-9

13. Carnaby G, Hankey GJ, Pizzi J. Behavioural intervention for dysphagia in acute stroke: a randomised controlled trial. Lancet Neurol. 2006; 5(1):31-7

14. Takahata H, Tsutsumi K, Baba H, Nagata I, Yonekura M Early intervention to promote oral feeding in patients with intracerebral hemorrhage: a retrospective cohort study. BMC Neurol. 2011; 11:6

15. Hagg M, Larsson B. Effects of motor and sensory stimulation in stroke patients with long-lasting dysphagia. Dysphagia 2004;
19(4):219-30

16. Babani N, Hattangadi GA. Bedside assessment protocol and grading scale for dysphagia in adults. Journal of Indian Speech Language \& Hearing Association 2014; 28(1):10-24

17. Crary MA, Carnaby-Mann GD, Groher ME. Initial psychometric assessment of a functional oral intake scale for dysphagia in stroke patients. Arch Phys Med Rehabil. 2005; 86:1516-20

18. Kahrilas PJ, Logemann JA, Krugler C, Flanagan E. Volitional augmentation of upper esophageal sphincter opening during swallowing. Am J Physio. 1991; 260:G450-6

19. Kahrilas PH, Logemann JA, Lin S, Ergun GA. Pharyngeal clearance during swallow: a combined manometric and videofluoroscopic study. Gastroenterology 1992; 103:128-36

20. Kahrilas PJ, Lin S, Logemann JA, Ergun GA, Facchini F. Deglutitive tongue action: volume accommodation and bolus propulsion. Gastroenterology 1993; 104:152-62

21. Fujiu M, Logemann J. Effect of a tongue-holding maneuver on posterior pharyngeal wall movement during deglutition. Am J Speech Lang Pathol. 1996; 5:23-30

22. Fujiu M, Logemann J, Pauloski B. Increased Post-operative posterior pharyngeal wall movement in patients with anterior oral cancer: preliminary findings and possible implications for treatment. Am J Speech Lang Pathol. 1995; 4:24-30

23. Gupta H, Banerjee A. Case Report Recovery of Dysphagia in Lateral Medullary Stroke.Hindawi Publishing Corporation, Case Reports in Neurological Medicine 2014; Volume 2014, Article ID 404871, 4 pages http://dx.doi.org/10.1155/2014/404871

24. K Govathi N, Akanksha G, Sushant B, Arun G. Recovery Pattern of Dysphagia in Lateral Medullary Infarction- A Case Report. GlobOto 2017; 3(2): 555610. DOI: 10.19080/ GJO.2017.03.555610

25. Rey-Matias R, Froilan C, Leochico D. Rehabilitation Techniques in Dysphagia Management among Stroke Patients: A Systematic Review. Int J Phys Med Rehabil. 2016; 4:340. doi:10.4172/2329-9096.1000340

26. Smithard DG, O'Neill PA, England RE, Park CL, Wyatt R, Martin DF, et al. The natural history of dysphagia following a stroke. Dysphagia 1997; 12(4):188-93

27. Waxman MJ, Durfee D, Moore M, Morantz RA, Koller W. Nutritional aspects and swallowing function of patients with Parkinson's disease. Nutr Clin Pract. 1990; 5:196-9

28. Shaker R, Kern M, Bardan E, Arndorfer RC, et al. Effect of isotonic/isometric head lift exercise on hypopharyngeal intrabolus pressure. Presented at the Fifth Annual Dysphagia Research Society Meeting [abstract]. Dysphagia 1997; 12:107

29. Shaker R, Kern M, Bardan E, Taylor A, et al. Augmentation of deglutitive upper esophageal sphincter opening in the elderly by exercise. Am J Physiol. 1997; 272:G1518-22. 